\title{
Heroes, Gods and Demons in the Religious Life of Akrai (Sicily) in Hellenistic Age•
}

\author{
Paolo Daniele Scirpo
}

Post-doc researcher in Classical Archaeology, National and Kapodistrian University of Athens (Greece) pascirpo@arch.uoa.gr

\section{Doi:10.5901/ajis.2015.v4n1p479}

\section{Abstract}

Entrusting the passage of souls on the other side of Acheron, people places in the ancient tombs a donation for the "ferryman" and a funeral wealth that could somehow serve the deceased in his eternal abode in Hades. Among the most common, as well as pottery, can be found anthropomorphic and zoomorphic clay statuettes, linked by a direct thread to the sphere of the divine. In this paper, we want to pay attention on the small Akrai, sub-colony of Syracuse, where since its founding in the second quarter of the 7th century BC, the religious life of its inhabitants in pursuit of peace, under the watchful eye and detached of the Olympian pantheon, was focused to make benevolent the souls of the dead or to exorcise the evil forces that in the mouth of poets, had taken the monstrous form notes in mythology. The phenomenon of the heroic cult in Akrai had found, especially in the Hellenistic age, a not indifferent political support, in the person of Hiero II, king of Syracuse. The stone quarry (latomeion) on the south-eastern of Acremonte, already active in the late 6th century BC, changes destination towards the end of Agathocles's kingdom (4th century BC), when we assist its transformation into a worship place whose evidence has also inspired the scholarly name "Templi Ferali", perhaps attributable to Paolo Orsi. Some materials, finally, from the Judica Collection and from stores of the Archaeological Museum in Syracuse, allow us to glimpse the divine and monstrous forms who, as glimmers of light in the darkness that still reigns on our knowledge of the religious sense and beliefs of Siceliotes, accompanied the earthly journey of the Acrenses.

Keywords: Akrai, cult, Heroes, Gods, Demons

\section{Introduzione}

Nell'affidargli il passaggio delle anime sull'altra riva dell'Acheronte, gli antichi riponevano nelle tombe un obolo per il traghettatore Caronte ed un corredo funebre che potesse in qualche modo servire al defunto nella sua eterna dimora nell'Ade. Tra gli oggetti più comuni, oltre la ceramica, si possono ritrovare statuette fittili antropomorfe e zoomorfe, legate da un fil rouge alla sfera del divino.

In questo contributo, si vuole porre attenzione sulla piccola Akrai $^{1}$, sub-colonia di Siracusa ${ }^{2}$ (fig. 1), dove fin dalla sua fondazione nel secondo quarto del VII secolo a.C. ${ }^{3}$, la vita religiosa dei suoi abitanti in continua ricerca della serenità, sotto lo sguardo vigile e distaccato del pantheon olimpico, era focalizzata a rendere benevoli le anime dei morti 0 ad esorcizzare quelle forze del male che nella bocca dei poeti, avevano assunto le sembianze mostruose note alla mitologia.

Nilsson distinse nella religione greca due differenti ed opposte correnti di pensiero, nate per il medesimo scopo, cioè quello di consolare l'umanità nel difficile cammino terreno. La prima aspirava all'unione col divino, tramite il misticismo e l'estasi, sotto la guida del più rivoluzionario degli dei dell'Olimpo, Dioniso. La seconda invece, preferiva meritarsi la pace e la serenità, ubbidendo a severe norme comportamentali dettate dalla divinità (Cfr. Nilsson, 1940

\footnotetext{
- Il presente contributo è la versione aggiornata della comunicazione fatta al convegno «Sulle Rive dell'Acheronte. Costruzione e Percezione della Sfera del Post Mortem nel Mediterraneo Antico» (Velletri (RM), $12-16$ giugno 2012). Vorrei in questa sede ringraziare il Comitato organizzatore del convegno per avermi dato la possibilità di conoscere Velletri ed il Museo delle Religioni, intitolato a Raffaele Pettazzoni. Sono inoltre grato verso il Museo Archeologico Regionale "Paolo Orsi" di Siracusa nelle persone della sua direttrice Beatrice Basile, e delle dott.se Angela Maria Manenti e Giuseppina Monterosso per avermi fornito il materiale fotografico dei pezzi presentati e per le ricerche d'inventario. Non posso infine, non ricordare in questa sede anche l'Istituto di Studi Acrensi di Palazzolo Acreide, nella persona del suo direttore, prof. Salvatore Distefano.

1 Cfr. Judica 1819; Schubring 1867; Hülsen 1893; Bernabò Brea 1956, 1986; Tusa 1958; Ziegler 1964; Voza 1971, 1973, 1976, 1980a, 1980b, 1999; Voza \& Lanza 1971-1994; Scirpo 1996-2004, 2004, 2005, 2007, 2005-2013; Guzzetta 2002; Distefano 2006.

2 Per una bibliografia completa ed aggiornata, cfr. Ampolo 2011.

${ }^{3}$ Sulla penetrazione siracusana in territorio siculo, cfr. Di Vita 1956. Contra Greco \& Torelli 1984.
} 
[2006: 100]).

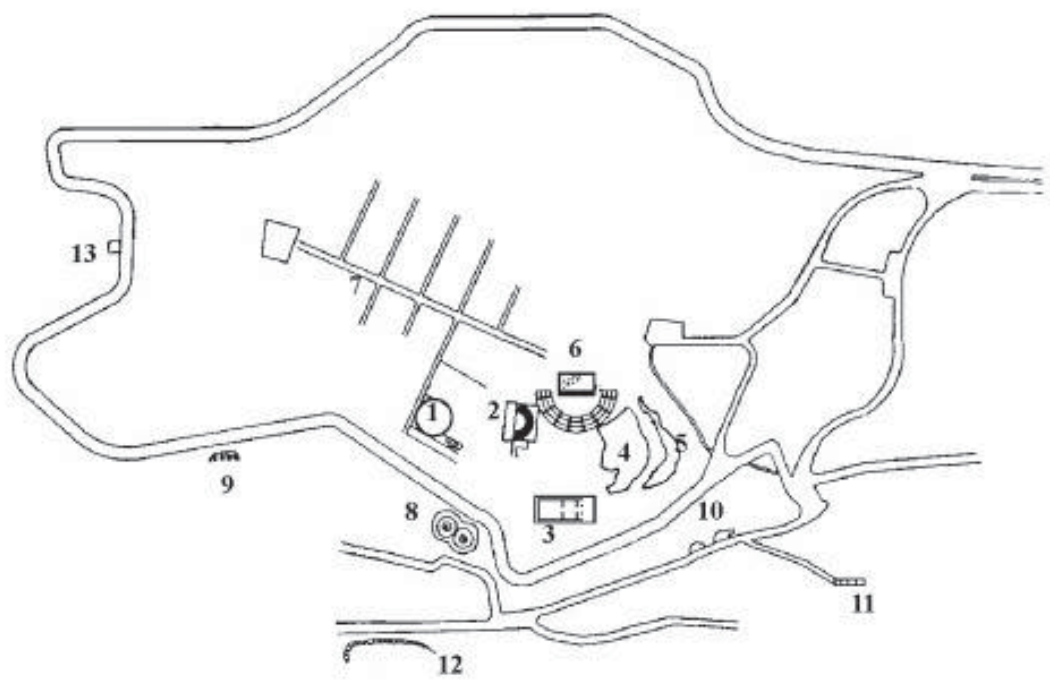

Fig. 1 - Il sito archeologico di Akrai (Scirpo 2004, fig. 6)

Già in età classica, i Greci differenziavano quattro categorie di esseri (Dèi, Dèmoni, Eroi e Uominii $)$ dei quali solo i primi tre erano protagonisti insieme agli Inferi dei racconti mitici5.

\section{Eroi}

Legato alla sfera pubblica, il fenomeno del culto eroico ha trovato ad Akrai, soprattutto in età ellenistica un supporto politico, non indifferente nella persona di lerone II, re di Siracusa6.

La latomia posta sul versante sud-orientale dell'Acremonte (Cfr. Bernabò Brea, 1956: 73-88, figg. 21-28, tavv. XIII-XVI), già attiva alla fine del VI secolo a.C. (Cfr. Laviosa in Bernabò Brea, 1956: 88), dalla quale fu probabilmente tratto il materiale per l'edificazione dell'Aphrodision arcaico (Cfr. Bernabò Brea, 1986), cambiò destinazione verso la fine del regno di Agatocle (316-289 a.C.), quando si assistette alla sua trasformazione in luogo di culto le cui evidenze hanno inoltre ispirato la denominazione dotta di «Templi Ferali», forse attribuibile a Paolo Orsi (fig. 2).

${ }_{4}^{4}$ Plat., Crat., 397 C. Per questo schema tetradico nei testi letterari/filosofici e nella religione popolare, cfr. Sfameni Gasparro 1997: $70-$ 71.

5 Plat., Resp., III, 392 A.

${ }^{6}$ Cfr. De Sensi Sestito 1977, Distefano 2009 e più in particolare sulla kulturpolitik del sovrano, Lehmler 2005. Per la sua politica religiosa, cfr. Bell 1999 ed in particolare per Akrai, cfr. Scirpo 2010 (c.d.s.).

${ }^{7}$ Lo stesso toponimo della contrada «dei Santicelli» usata da Judica (1819: 118) si ritrova anche in Kaibel 1890 (IG, XIV) ma Paolo Orsi (1889) fa cenno alla denominazione tradizionale di «Templi Ferali», sebbene preferisca usare il termine scientificamente più corretto di Heroa. Anche Biagio Pace (1945: 515) riferendo meccanicamente la vecchia denominazione, fornisce (p. 518) alcune spiegazioni del culto ivi celebrato. 


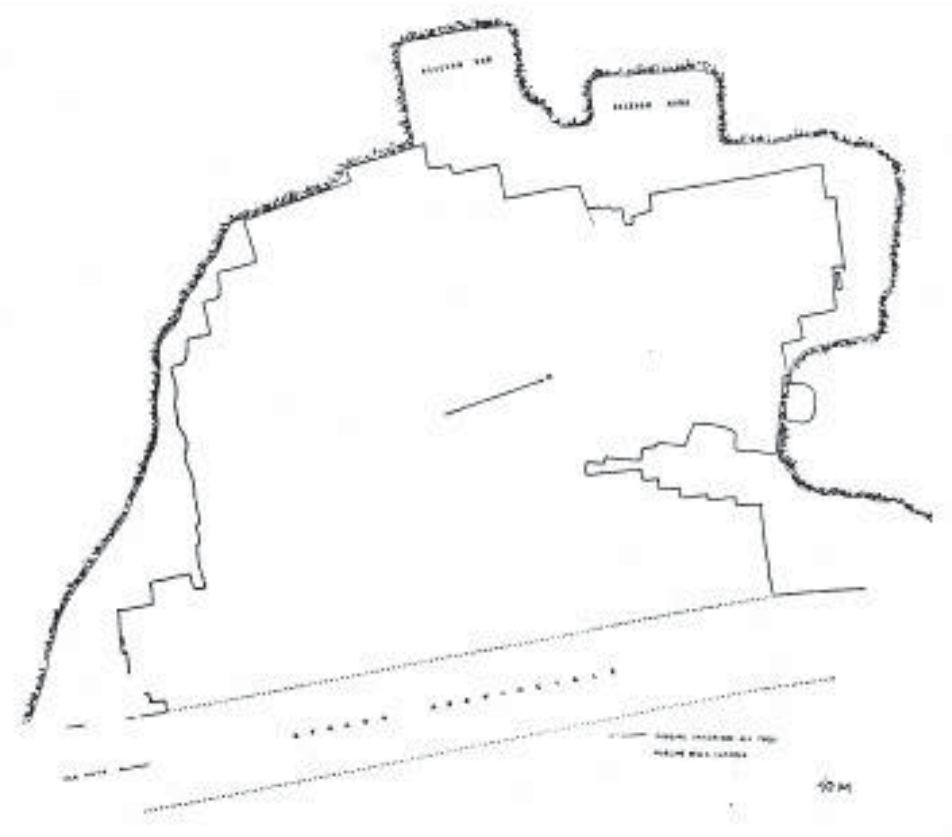

Fig. 2 - Pianta della latomia extraurbana i c.d. "Templi Ferali" (da Bernabò Brea 1956, fig. 21)

Santuari simili sono presenti in altre poleis siceliote sia appartenenti al regno di lerone (Siracusa ${ }^{8}$, Naieton ${ }^{9}$, Megara Hyblaia ${ }^{10}$, Akrillai11 ${ }^{11}$ Morgantina ${ }^{12}$ ) che da esso dipendenti (Enna $\left.{ }^{13}\right) 0$ alleate (Akragas $\left.{ }^{14}\right)$.

Dopo gli scavi condotti nel 1953 da Clelia Laviosa (Cfr. Laviosa in Bernabò Brea, 1956: 81-88), l'immagine che si ha dell'area permette di aver un'idea più chiara di questo santuario. Oltre a quelle dei due recessi (Nord e Sud) già noti al barone Gabriele Judica ${ }^{15}$, si è accertato che tutte le restanti pareti della latomia erano interessate da piccole cavità di forma per lo più rettangolare, a volte con frontoncino e spesso accompagnate da iscrizioni incise ${ }^{16}$. Al loro interno, vi erano deposte le immagine sacre dei defunti o sotto forma di dipinti sullo stesso fondo roccioso o su legno o sotto quella ben più comune per la zona, di rilievi in calcare. Due grandi fosse ai piedi delle pareti dei due recessi inoltre, hanno restituito materiale ceramico di uso sacro, depositato dopo i sacrifici, tra le tracce di bruciato.

I rilievi potevano appartenere a due categorie: la prima che mostra una scena di simposio in onore di defunti

${ }^{8}$ Cfr. Orsi 1904: 276-280.

${ }^{9}$ Cfr. Orsi 1897: 82-87; La Rosa 1988-1989, 1993.

${ }^{10}$ Cfr. Orsi $1897: 87$.

11 Cfr. Distefano G. 2000.

${ }_{12}$ Per un rilievo funebre in calcare databile alla fine del III secolo a.C., con heros equitans, cfr. da ultimo Germanà Bozza 2008: 6, fig. 16 .

${ }^{13}$ Cfr. Orsi 1931: 382-383, figg. 6-8.

14 Cfr. De Miro 1966, 1981.

${ }^{15}$ Laureato in Legge, il barone Gabriele Judica (1760-1835) non esercitò mai la carriera forense per dedicarsi alla ricerca storica ed archeologica. Ricopri la carica di Regio Custode della Antichità del Distretto di Noto. Con i reperti archeologici scoperti ad Akrai costitui un Museo privato che fu oggetto di visite da parte dei viaggiatori italiani ed europei. Per le vicende della collezione Judica, cfr. Agnello 1965, Lombardo 1998 e da ultimo Musumeci 2009. Sulle testimoniane degli archeologi tedeschi suoi ospiti, cfr. Blanck 2002-2003: 314$315,318,323$.

${ }^{16}$ Pace (1945: 510-517) ha riconosciuto nell'adozione delle dediche rupestri un costume tipicamente siculo, dove la venerazione delle Dee della natura e dell'Oltretomba (Demetra e Kore) si associava a quella verso il loro corteggio di ninfe (Paides, Meteres) come mostrerebbe il santuario rupestre di Buscemi. Scettico però sul carattere ctonio di questo santuario extraurbano di Akrai, appare Pugliese Carratelli (1951: 75). 
eroizzati, sdraiati su klinai17, la seconda, invece, quella del cosiddetto heros equitans (Cfr. Machaira, et alii 1992), ovvero la raffigurazione di un cavaliere al galoppo e in quiete, accolto da servi e familiari.

Dei sei rilievi editi18 però, solo due sono stati rinvenuti nella latomia: il primo, in frammenti, da Judica ${ }^{19}$ ed il secondo, integro ed in situ, da Laviosa ${ }^{20}$ (figg. 3-4).

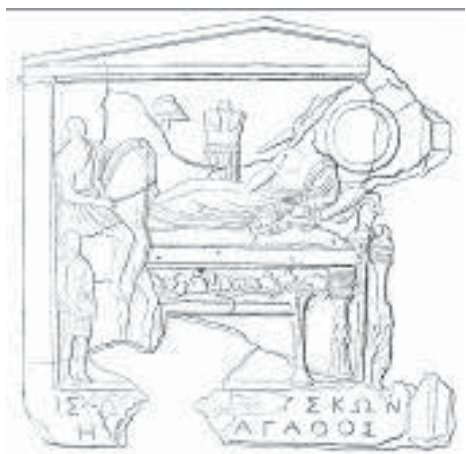

Fig. 3 - Rilievo votivo dei due heroes (da Judica 1819, tav. XIV.1)

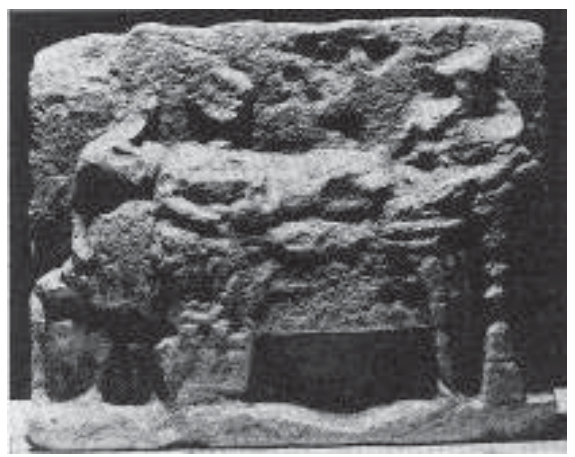

Fig. 4 - Rilievo votivo del banchetto funebre (da Bernabò Brea 1956, tav. XXXII.4)

Se il primo sopra l'invocazione in onore di «Zẃmupos» ${ }^{21}$, con l'immagine di due heroes agathoi (Is[tiodoros?] e [F]yskon) da l'impressione che essi appartengano alla casta militare della piccola polis, il secondo mostra chiaramente l'heros banchettante alla tavola di Hades, con accanto una donna seduta ai piedi della kline ed un giovane servo.

17 Per l'interpretazione delliconografia, cfr. Guarducci 1962. Per l'origine campana del motivo figurativo dell'heros equitans, cfr. Germanà Bozza 2008. Una nuova analisi iconografica è proposta da Portale (2010) dove si ritiene che l'associazione del motivo dell'eroe banchettante e del cavaliere sia di derivazione asiatica e possa spiegarsi con la tendenza dell'elites provinciali di rimarcare con ridondanza le proprie origini greche in un periodo come il II secolo a.C. che vedeva l'isola sotto il dominio di Roma.

18 Per una recente esamina d'insieme dei rilievi, databili sulla base dei confronti con esemplari micrasiatici al II secolo a.C., cfr. Portale 2010: 56-63.

${ }^{19}$ Rinvenuto nell'autunno 1817 nel recesso Sud dei «Templi Ferali» (Coll. Judica $2268=$ MAR Sr 2273). Cfr. Judica 1819: 119, tav. XIV,1. Bernabò Brea 1956: 147, n. 12, tav. XXXI, 1.

${ }^{20}$ Rinvenuto in situ nel 1953 nella parete Nord della latomia (Antiquarium di Palazzolo Acreide). Cfr. Bernabò Brea 1956: 148, n. 16, tav. $X X X I I, 4$ e XV, 1-2.

${ }^{21}$ Nome comunissimo ad Akrai e nel regno di lerone II, come testimonia anche quello di un omonimo olimpionico di Siracusa, nella corsa dello stadion nel 220 a.C. Cfr. Stampolidis \& Tasoulas 2004. 
Gli altri quattro rilievi22, tre dei quali23 sono stati trovati da Judica nel 1817 sul piano d'Acre (ovvero in una zona non meglio identificata dell'area urbana) in un vano forse privato, probabilmente di carattere sacro ${ }^{24}$, sono tutti comunque databili sulla base stilistica al III secolo a.C.

II Brelich ha fatto notare come «Con il diffondersi dell'eroizzazione dei morti comuni, uno dei più frequenti modi di raffigurazione del defunto eroizzato diventa quello del cacciatore, a cavallo, con il cane: spesso, anzi, sono il cavallo ed il cane che con la loro presenza accanto all'eroe (raffigurato in atto di banchettare) lo definiscono come cacciatore; diventando eroe, il defunto diventa eo ipso cacciatore, perché l'eroe è cacciatore» (Cfr. Brelich, 2010 : 148).

Grazie alle descrizioni tratte da alcuni passi dei tragediografi25 ed alla ceramica cronologicamente omogenea che è stata rinvenuta nelle fosse scavate sul fondo roccioso di fronte alle edicolette (Cfr. Laviosa in Bernabò Brea, 1956: pp. 86-87) (fig. 5), è possibile ricostruire a grandi linee i riti celebrati (Cfr. Burkert, 1977 [1993: 164-170]; 1979 [1997: 80]; Simon 2004) (forse anche di notte) in onore di questi defunti eroizzati (Cfr. Elroth, 1992; Antonaccio, 1995; Machaira, 2000): una processione partita dal centro cittadino, si dirigeva dapprima verso la valle dove una fonte forniva l'acqua necessaria ai ritij2 ${ }^{2}$, poi verso la ex latomia. Lì, dopo aver bruciato un'offerta votiva nella fossa, nel momento culminante siglato da un urlo estatico (ołołuyŕ), si spegneva il fuoco versando (Xoń) vino conservato nei piccoli crateri, e si spalmava d'olio e miele i naiskoi nelle pareti.

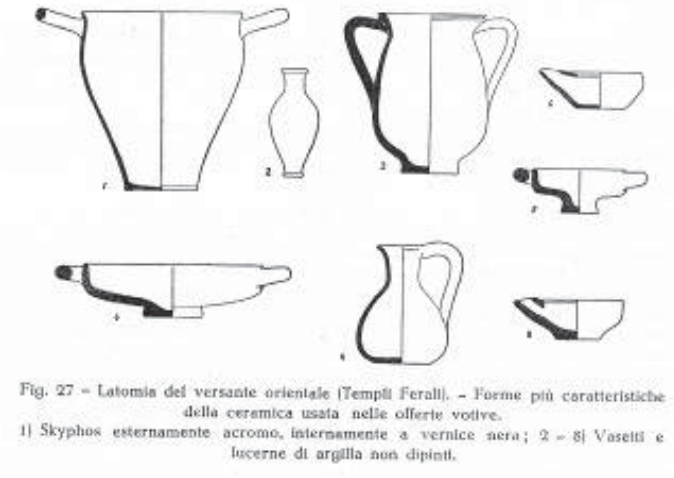

Fig. 5 - Forme ceramiche rinvenute nelle offerte votive

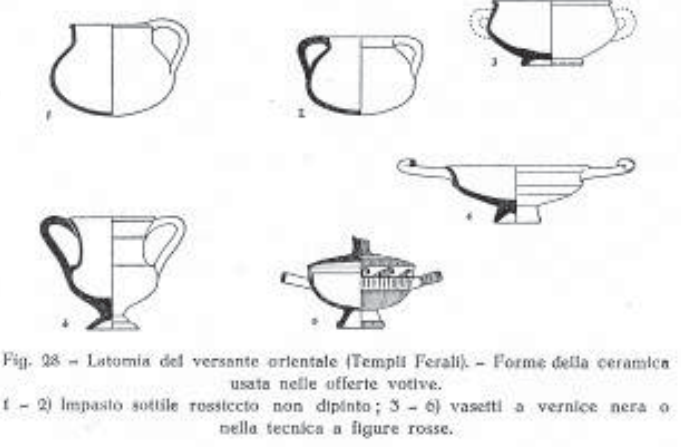

(da Bernabò Brea, 1956, figg. 27-28)

Ma chi erano i defunti qui adorati?

Incisa su una tavoletta di piombo (fig. 6), edita da Manganaro (Cfr. Manganaro, 1997: 310-318) e datata sulla base paleografica proprio al III-II secolo a.C., una lista di 52 nomi27, composta forse dalle leve di un lochos di caduti acrensi, potrebbe essere la testimonianza mancante sull'attività di reclutamento del suo esercito di cittadini che lerone II, demonizzando l'uso di mercenari, instaurò nel suo regno. Come centro di addestramento militare (Cfr. Gauthier, 1995: 9) non immune da una sorta di divinizzazione del sovrano (Cfr. Consolo Langher, 2000), il ginnasio, la cui presenza anche

22 II quarto rilievo, frammentario, mostra solo una trapeza ed un bomos, senza permettere una sua lettura migliore. Cfr. Bernabò Brea 1956: 148, n. 17, tav. XXXIII, 8.

${ }^{23}$ II rilievo (Coll. Judica 2263) porta un'iscrizione frammentaria (Heros Agathos) mentre il rilievo (Coll. Judica 2262), sebbene illustrato da Judica (1819, tavv. XIV, 2-3 e XVI, 2), è sfuggito all'attenzione di Bernabò Brea (1956: 147-148, nn. 13, 14, 15, tavv. XXX, 2 e XXXI, 2).

${ }^{24}$ Nasce il sospetto che il vano quadrangolare $(m .3$ di lato) con pavimentazione a mosaico (recante al centro la scritta EP $\Omega$ ) possa trovarsi nei dintorni dell' «Agorà degli Dei», anche se è difficile stabilire esattamente il luogo. Sulle agorai di Akrai in età ellenistica, cfr. Scirpo 2011.

${ }^{25}$ Aesch., Pers., 607-622; Choeph., 84-164; Soph., Oed. Col., 466-492.

${ }^{26}$ A poca distanza dai «Templi Ferali» si trova una fonte d'acqua che insieme a quella c.d. della Madonna delle Grazie nei pressi dei «Santoni», potrebbe aver assolto al fabbisogno nella prima fase di vita della polis, e dopo la costruzione dell'acquedotto sotterraneo cittadino, essersi limitato all'uso sacro. In alternativa, potrebbe anche ipotizzarsi l'uso di una fonte nella stessa latomia, unica traccia della quale sarebbe un cunicolo (mai esplorato) citato dallo Schubring e visto da Bernabò Brea (1956: 57) che lo reputa «lo sbocco di uno dei cunicoli dipartentesi dal fondo di qualche profondissimo pozzo del sovrastante piano».

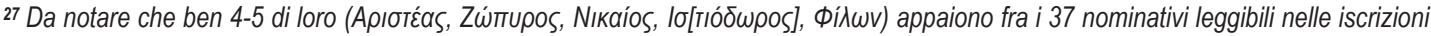
dei «Templi Ferali». 
ad Akrai28 è testimoniata da un'epigrafe (Cfr. Judica, 1819, tav. III, 4; CIG, n. 5429; IG, XIV, n. 213; S.E.A., n. 10, p. 158) potrebbe davvero costituire il primo tassello del processo di eroizzazione della casta militare (la c.d. Gioventù Ieroniana ${ }^{29}$ ) e di definitiva consacrazione al rango di polis della piccola Akraij3.

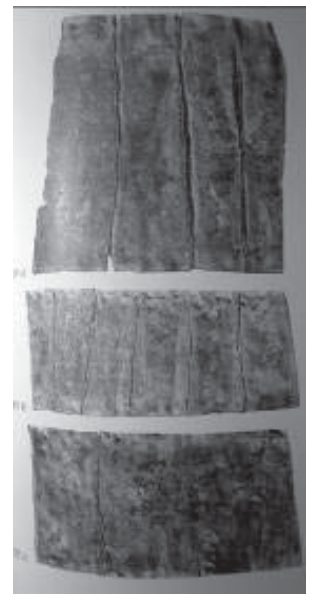

Fig. 6 - Tavoletta di piombo (da Manganaro 1997)

In effetti, solo dopo la grande impresa di Alessandro Magno, la religiosità ellenica prese coscienza di molte credenze orientali. Una di esse fu certamente quella dell'Agathos Daimon del sovrano, del condottiero (Cfr. Ross Taylor, 1927, 1930). Da qui, all'attribuire la dimensione eroica anche agli spiriti dei soldati caduti in battaglia il passo fu breve.

Dai dati raccolti, si reputa il culto dei defunti sia iniziato durante l'ultima fase del regno di Agatocle e che fosse continuato ininterrottamente almeno fino al II secolo a.C. ${ }^{31}$.

I «Templi Ferali» quindi, potrebbero così essere il cenotafio ${ }^{32}$ dei caduti acrensi nelle campagne militari condotte successivamente da Agatocle ${ }^{33}$, Iceta, Pirro, lerone e leronimo, dove si celebravano forse anche in un mese dedicato al

${ }^{28}$ Fra $i$ ventuno siti in Sicilia dove è attestata (per lo più epigraficamente) la presenza di un ginnasio, ben otto di essi (Akrai, Heloros, Leontinoi, Megara Hyblaia, Morgantina, Neaiton, Syrakousai e Tauromenion) appartenevano al regno di lerone II. Per l'istituzione della Ginnasiarchia, cfr. Cordiano 1997 e sulla sua distribuzione in Sicilia, cfr. Prag 2007: 87-96.

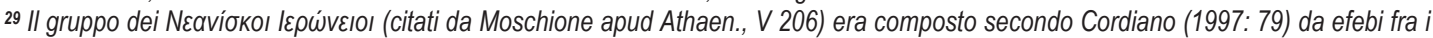
15 ed $i$ 17 anni.

30 Sui motivi di questa evoluzione per certi versi naturale che appare diffusa in tutta la Sicilia, cfr. La Torre 2008. Nel caso di Akrai non è escluso che abbia avuto un ruolo la possibile origine acrense dello stesso lerone II. Cfr. Distefano 2009 e Scirpo 2010.

31 In effetti, si sa dalle fonti che Agatocle si stesse preparando in quel periodo (299 a.C.) ad invadere l'Italia, con al seguito un esercito di circa 30.000 fanti e 3.000 cavalieri.

32 Un cenotafio o anche una dimora spesso presero il posto della tomba come condizione necessaria ma non sufficiente per l'istituzione del culto eroico. Cfr. Seiffert 2005. Secondo la Portale (2010: 62, nota 3) nei «Templi Ferali» vi fu una commistione delle tre forme di culto (eroico, funerario e degli antenati) dove si avverti spesso l'esigenza di denominare il defunto eroizzato con il proprio nome inciso.

${ }^{33}$ Dopo la sua morte (288 a.C.) e la successiva lotta per il potere da parte di Menone (sostenuto dalle armi puniche) per impadronirsi del potere, fu Iceta che sostenuto dal popolo, ristabili una nuova tirannia (288-279 a.C.). Questi, sconfitto dal tiranno di Akragas, Finzia, nei pressi di Hybla e dai Cartaginesi, fu esiliato da Tinione che assunse la tirannia assieme a Sosistrato (279-277 a.C.). La susseguente guerra civile scoppiata fra $i$ due e la contemporanea aggressione punica fecero si che $i$ due contendenti chiamassero in aiuto Pirro, lasciando nelle sue mani le sorti della Pentapoli. Il re epirota non riusci nella sua breve permanenza sull'isola (277-275 a.C.) a realizzare il suo sogno di conquista e di scacciare i Punici. Uno dei suoi generali, lerone fu nominato (275 a.C.) nella neonata repubblica siracusana, stratega assieme ad Artemidoro. Per eliminare la minaccia dei mercenari Mamertini, dapprima li scacciò dalla città, poi li sconfisse in una battaglia sul fiume Longano (270 a.C.) nei pressi di Mylai. La battaglia vinta da lerone lo spinse all'assedio di Messana (265 a.C.) dove i Mamertini chiesero l'aiuto dapprima di Cartagine e poi di Roma. L'inizio della susseguente prima Guerra Punica (264241 a.C.) vide cosi lerone costretto a scegliere Roma (263 a.C.) come alleata ed a rimanerle fedele fin dopo lo scoppio della seconda Guerra punica (218-202 a.C.), quando alla sua morte (215), il successore al trono, il nipote leronimo, sobillato dal partito filo-punico ed accecato dalle vittorie di Annibale, spezzò l'alleanza siglata dal nonno e condannò la polis all'assedio romano (212 a.C.) sotto il comando del console Marco Claudio Marcello. 
culto dei morti (Nekysios?) ${ }^{34}$ delle cerimonie pubbliche di cordoglio e commemorazione ${ }^{35}$. Cosa che potrebbe spiegare l'enorme strato di ceneri rinvenuto nel 1953 dalla Laviosa, che non si giustificherebbe con la frequenza saltuaria di commemorazioni private.

Prendendo in esame solo superficialmente la categoria degli Eroi, la cui definizione non è qui d'uopo scandagliare, la nostra indagine ci porta dal figlio di Zeus e di Alcmena (Cfr. da ultimo, Stafford, 2012).

In una polis dorica, per quanto piccola che sia, non poteva infatti, mancare il culto di Herakles ${ }^{36}$, considerato e non a torto, in molti casi come il capostipite dei fondatori (Cfr. Icard-Gianolio, 2000) e durante il suo girovagare in Occidente, fondatore lui stesso di culti e città ${ }^{37}$.

Egli doveva avere avuto un posto di riguardo nel pantheon della metropoli ed un suo culto è certamente attestabile fin dal V secolo a.C., come testimoniano sia i dati della tradizione letteraria ${ }^{38}$ sia quelli che si ricavano da documentazioni archeologiche, come ad esempio, l'iscrizione ${ }^{39}$ a lui (Kraterophron) dedicata nel VII cuneo del teatro della Neapolis siracusana ${ }^{40}$.

Sebbene nessuna fonte antica, in primis Tucidide ${ }^{41}$, abbia fornito il nome dell'ecista di Akrai, sul cui status di subcolonia si potrebbe ancora discutere (Cfr. Lombardo, 2006 e Copani, 2008), di recente, Salvatore Distefano ha proposto di identificarlo come un membro del ghenos degli Heraklides ${ }^{42}$ sulla base della alta frequenza del teonimo nel ricco onomatologio acrense ${ }^{43}$, e di riconoscerlo nella figura campeggiante su di un rilievo rupestre di grandi dimensioni, sulla parete settentrionale della latomia urbana dell'Intagliatella ${ }^{44}$ (fig. 7).

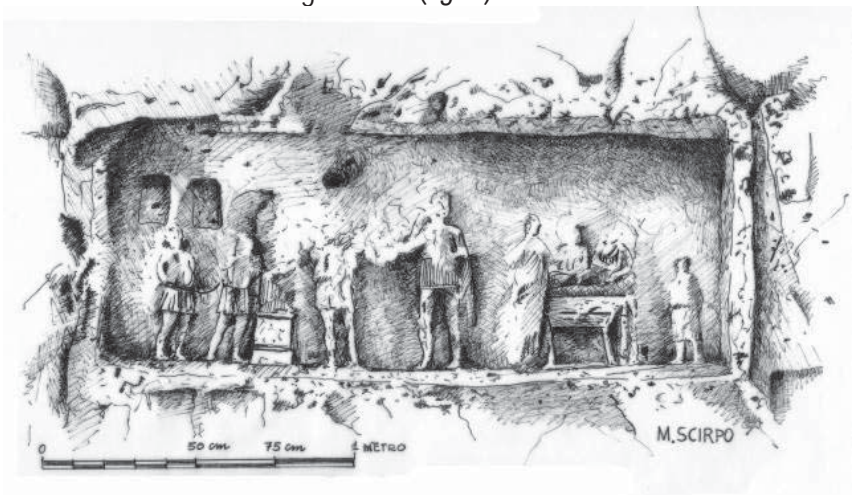

Fig. 7 - Rilievo rupestre con scena di sacrificio nella parete settentrionale della latomia dell'Intagliatella (disegno di Massimo Scirpo)

${ }^{34}$ Cfr. Trümpy 1997: 88 (etimologia del mese), 193 (attestato a Knossos).

${ }^{35}$ L'esempio più noto di commemorazione funebre militare è quella tramandata da Plutarco (Arist., 21) per i caduti di Platea. Cfr. Burkert 19972 [2011: 127-129].

${ }_{36}$ Per una sintesi sulla questione scaturita dalla testimonianza di Erodoto (II, 43, 44) sulla doppia natura (divina ed eroica) del suo culto, cfr. Lévêque \& Verbanck-Piérard 1989.

${ }^{37}$ A lui sono attribuite le fondazioni dei culti di Hera a Kroton, della ninfe Kyane a Syrakusai, nonché la paternità delle poleis di Kroton, Lokroi, Eryx. Cfr. Jourdain-Annaquin 2006. Per le origini rodio-cretesi del culto di Herakles a Gela ed Akragas, cfr. Scirpo 2014.

${ }^{38}$ Alla testimonianza di Tucidide (VII, 73,2) su una festa in onore di Herakles, fa seguito quella di Plutarco (Nic., XXIV,6) che menziona un Herákleion da cercare lungo la costa occidentale del Porto Grande in un punto rialzato del terreno. Una sua identificazione è proposta da Polacco \& Mirisola 2005.

${ }^{39}$ CIG 5369; IG XIV, 3; SEG XXXIV, 975; Di Martino 2005: 110.

${ }^{40} \mathrm{Da}$ non dimenticare, la sua raffigurazione in conii di IV secolo a.C. e la piccola statuina in marmo, conservata al MAR Paolo Orsi (n. 30575) di chiaro influsso lisippeo, databile agli inizi del III secolo a.C. cfr. Bernabò Brea 1958: 58 e da ultima Portale in Pugliese Carratelli 1996: 745, n. 358.

41 Thuc., VI, 5, 2-3.

42 Del quale faceva parte anche Archias, l'oikistes di Siracusa. Thuc., VI, 32. Strab., VI, 2,4.

${ }^{43}$ Alla già ricca silloge [S.E.A.] edita da Pugliese Carratelli in Bernabò Brea 1956, si devono aggiungere anche le tavolette di piombo inscritte pubblicate da Manganaro (1997: 310-318) che contengono preziosissimi particolari sulla suddivisione tribale e in demoi di Akrai negli anni del regno di lerone II.

${ }^{44}$ Cfr. Distefano 2006: 24-26. Per una diversa interpretazione del rilievo rupestre, cfr. Scirpo 2010 e Portale 2010: 55, 63. 
Come prova tuttavia di un culto direttamente dedicato all'eroe (piuttosto che al dio), si possono portare a testimoni alcuni reperti rinvenuti dallo Judica: una statuetta fittile dell'eroe con leontè nei pressi del pozzo nell'atrio del convento dei Padri Minoriti Osservanti di Santa Maria del Palazzo45, ed una gemma in agata con la testa dell'eroe sempre con leonté (Cfr. Judica, 1819: 106 e 152) (figg. 8-9).

Infine, la scoperta nella zona retrostante il bouleuterion di un frammento di statua marmorea di Herakles (la mano sinistra che trattiene la leontè) non fa che confermare il carattere civico del culto (Cfr. Distefano, 2006: 27, nota 58) che in tutta l'isola in generale mostra degli aspetti ctoni ed apotropaici che lo resero il soggetto ideale delle preghiere dei Sicelioti (Cfr. ad esempio, Manganaro, 2005).

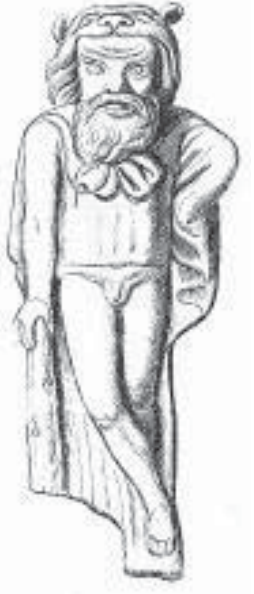

Fig. 8 - Statuetta fittile di Herakles (da Judica 1819, tav. X.4)

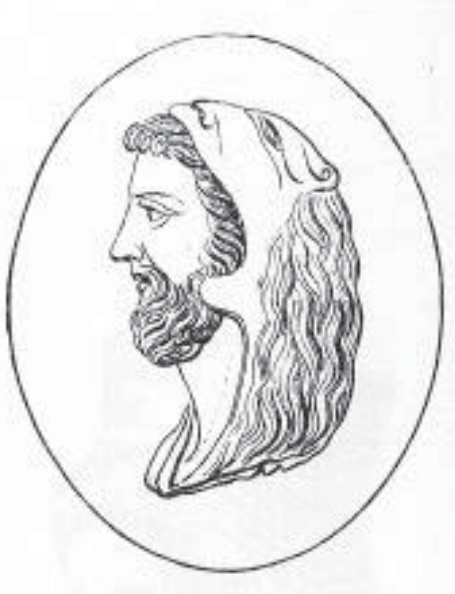

Fig. 9 - Gemma in Agata con profilo di Herakles (da Judica 1819, tav. XXIII.3)

Prototipo dell'eroe per eccellenza (Cfr. Brelich, 2010: 159-161), con una triplice funzione (Cfr. Cuartero i lborra, 1998) di liberatore dai mali e dalla tirannide, di fondatore di culti e poleis e di vincitore infine della morte ${ }^{46}$ con la sua finale apoteosi, Herakles, eroe-dio, fu visto come un modello eroico da imitare ${ }^{47}$.

\section{Dei}

Alzando lo sguardo verso l'Olimpo, la nostra attenzione si pone sui suoi abitanti che avevano un legame con il mondo degli Inferi.

Come è noto fin da Omero48, Hermes sulle cui origini s'è dibattuto da Nilsson in poi (Cfr. Nilsson, 1940 [2006: 6-7]; 1949 [2008: 121-122]; Otto 1947 [2004: 110-131]; Kerényi, 1944), è rappresentato come messaggero degli Dei ed allo stesso tempo, tramite tra il mondo dei viventi e quello dei morti. In qualità di «psychopompos», il dio ha il compito di scortare le anime dei defunti verso l'ingresso dell'Ade (Cfr. Raingeard, 1935).

Ad Akrai, la sua presenza è manifestata da pochi ma interessanti indizi:

45 /l convento, edificato a detta del Bonanni sui ruderi del c.d. Palazzo di lerone, che aveva al centro del chiostro un pozzo di età greca utilizzato dallo Judica come punto di riferimento per i suoi scavi, fu distrutto dal sisma del 1693 e riedificato più a valle, con lo stesso materiale di costruzione. Ciò ha cancellato anche le tracce dei ruderi antichi inglobati nell'edificio. Cfr. Bernabò Brea 1956: 27.

${ }^{46}$ Omero (II., V, 395-ss.) fa cenno alla lotta di Herakles con Hades nella quale il sovrano degli Inferi è ferito. Nell'Alcesti di Euripide, l'eroe vince Thanatos in persona per salvare la regina, mentre per compiere una delle sue fatiche, la cattura di Cerbero, aiutato da Hermes ed Athena, Herakles, iniziato ai Misteri Eleusini, scese all'Ade, dove tutte le ombre fuggono al suo cospetto tranne quella di Medusa, contro la quale si scaglia invano (Ps-Apoll., V, 12).

47 La Pedrucci (2009: 57, nota 113) fa notare come una monetina rinvenuta in una fossetta dei «Templi Ferali», porti come tipi monetali una testa femminile ed un leone, e propone di leggervi un legame con Herakles, piuttosto che con Cibele. Quale miglior offerta votiva per un defunto eroizzato che il simbolo dell'eroe per eccellenza!

${ }^{48} \mathrm{Hom}$., Od., XXIV. 
Lo Judica pubblicò una gemma in ametista dove è raffigurato il dio alato nell'atto di dare una serpe in pasto ad un ibis, uccello sacro e simbolo esso stesso del dio egizio Thot che in età ellenistica, fu identificato proprio con Hermes ${ }^{49}$ (fig. 10).

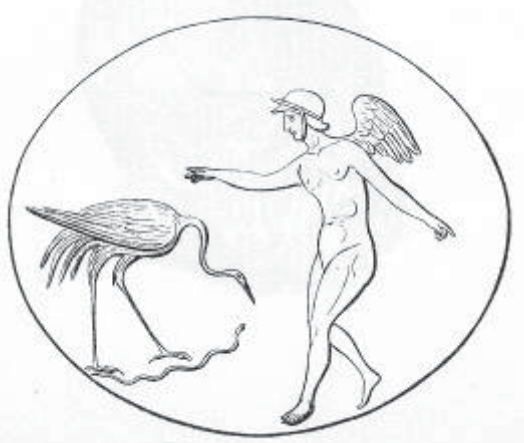

Fig. 10 - Gemma in ametista con Hermes (da Judica 1819, tav. XXXIII.8)

Come paredros subalterno («Kadmilos») (Cfr. Conze, 1880), il dio riconoscibilissimo grazie al tipico caduceo, si trova a fianco della dea Cibele nel rilievo II dei c.d. «Santoni»» ${ }^{50}$, assieme ad Attis ${ }^{51}$, ad Hecate ed ai Dioscuri52 (fig. 11).

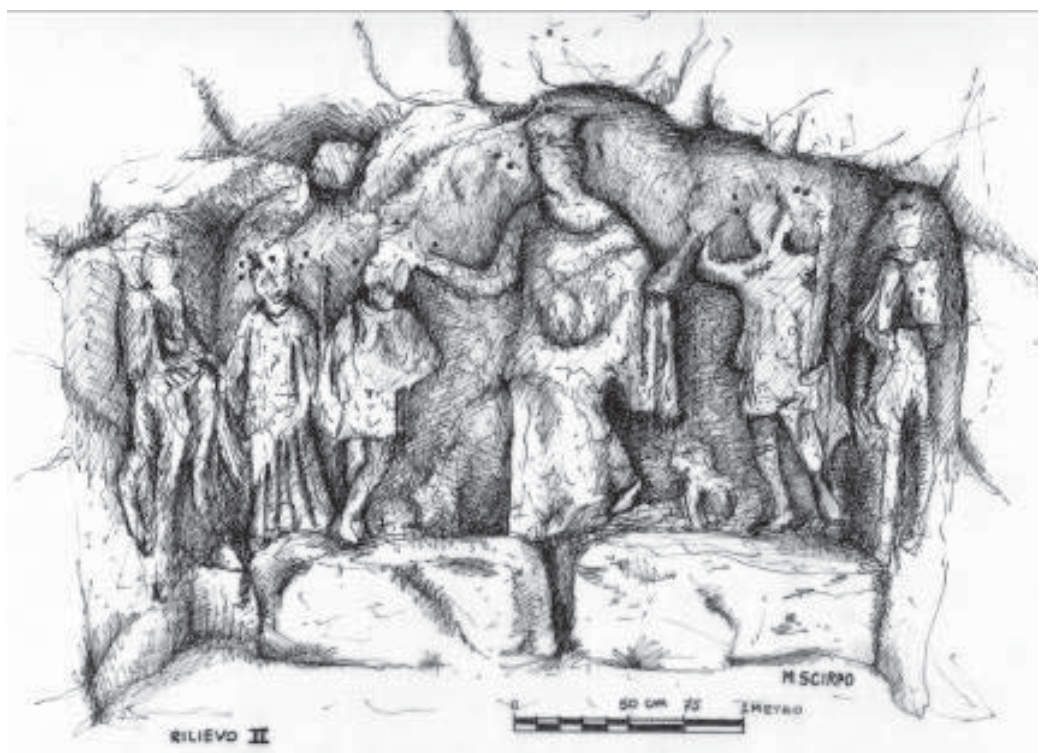

Fig. 11 - Rilievo II dei Santuario di Cibele ("Santoni”) (disegno di Massimo Scirpo)

${ }^{49}$ Cfr. Judica 1819: 158, tav. XXXIII, 8. Anche in una statuetta (fittile?) di personaggio maschile con pallio e capello, seduto e con un'asta in mano, potrebbe a nostro avviso riconoscersi le sembianze di Hermes (tav. VIII, 3).

50 Cfr. Bernabò Brea 1956: 89-111 (in particolare per il rilievo II: 94-97); Sfameni Gasparro 1973: 126-149; 1986, Scirpo 2007; 2015; Pedrucci 2009: 44-67 e 107-120.

51 Dal confronto col rilievo VIII, Bernabò Brea (1956: 96) preferisce riconoscervi il satiro Marsia.

${ }^{52}$ La presenza dei Dioscuri potrebbe a detta di Germanà Bozza (2008: 12-13), riflettere l'interesse della casta militare locale verso il culto della Dea Cibele. Sulla lettura del rilievo II, cfr. Pedrucci 2009: 51-52 e 57-58. 
Ed in questa veste, egli appare anche in un rilievo d'età alto imperiale, in calcare rinvenuto ad Akrai53 (fig. 12).

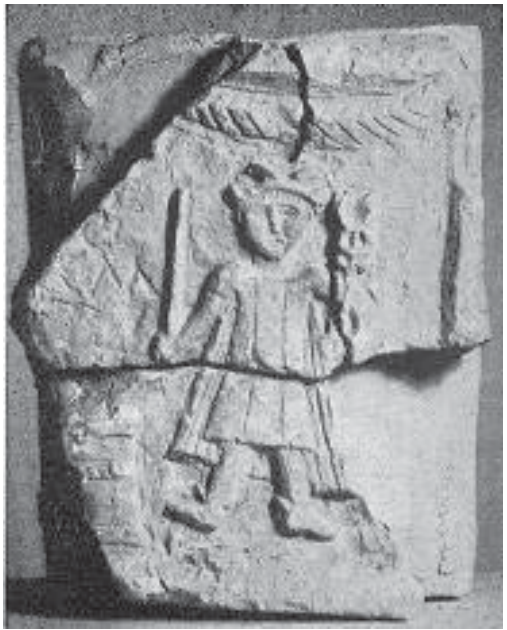

Fig. 12 - Rilievo in calcare con Hermes psychopompos (da Bernabò Brea 1956, tav. XXXII.3)

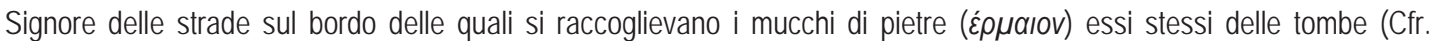
Nilsson, 1906: 388; 1940 [2006: 6-7]; 1949 [2008: 121-122]; 1950: 508), da cui il dio prese il suo nome ${ }^{54}$, egli fu guida divina, protettore dei viandanti e spirito della notte (Cfr. Otto, 1947 [2004: 119-126]).

A chi se non ad Hermes, la cui natura dolcemente crepuscolare (Cfr. Kerényi, 1952 [2001: 203]) affascinava l'animo umano, avrebbero potuto rivolgersi anche gli Acrensi nel loro difficile ultimo viaggio, nel regno dei morti che sono spesso raffigurati sotto forma di serpente.

\section{Demoni}

Della terza categoria di cui abbiamo fatto obbligo parlare, quella dei Demoni (Cfr. Burkert, 1977 [1993: 380-384]) è secondo il nostro avviso quella più affascinante dal punto di vista religioso.

Grazie ad alcuni materiali provenienti dalla collezione Judica e dai magazzini di Musei siciliani, si riesce a nostro avviso ad intravedere alcune delle forme divine e mostruose che, come spiragli di luce nell'oscurità che ancora regna sulla nostra conoscenza del senso religioso e delle credenze dei Sicelioti, accompagnavano il cammino terreno degli Acrensi.

La fede in una vita migliore sulla terra era alimentata anche dalla speranza di ottenerla grazie all'aiuto di divinità ctonie che in età ellenistica, ritornarono alla luce dopo i secoli della supremazia del pantheon olimpico.

Fra le iscrizioni incise sulle pareti dei «Templi Ferali» (Cfr. Pugliese Carratelli in Bernabò Brea 1956 [S.E.A., n. 22 (3) e (15)]), appare oltre all'invocazione dell'heros agathòs anche quella verso l'AvaӨós $\Delta \alpha i ́ \mu \omega v^{55}$, lo spirito protettore della casa, spesso rappresentato anch'egli come un serpente (Cfr. Nilsson, 1940 [2006: 67, 70], 1949 [2008: 135]) in onore del quale dopo il pasto, gli antichi erano soliti versare a terra delle gocce di vino puro dalla coppa quale offerta.

Ritenuto un "relitto" dell'antico culto di Dioniso prima che fosse accettato nel pantheon olimpico, l'Agathos Daimon, con la sua figura di essere ctonio a metà strada fra l'eroe ed il dio, rappresenta l'unica eccezione nel mondo dei demoni che al contrario, erano sostanzialmente privi di culto e di miti a loro riferiti56.

Raffigurato fin dagli inizi del V secolo, in vari modi (Cfr. Gansichinietz, 1918; Bermond Montanari, 1958; Durrand,

${ }^{53}$ MAR Sr n. 12243. Cfr. Bernabò Brea 1956: 149, n. 22, tav. XXXII, 3. Di recente la Kunz (2006: 73 e 197) lo data ad età alto imperiale anche a causa della sua finora criptica breve iscrizione in latino (C/EM).

54 Sebbene già presente nel miceneo e-ma-a2 (Py Tn 316), la sua etimologia appare incerta. Cfr. DEMGOL, s.v. Ermes.

${ }_{55}$ Già Platone (Resp., 469b, 540c) ritenne giusto che i caduti fossero adorati non come heroes ma come daimones.

${ }^{56}$ Esiodo (Erga, 122-126) narra come per volere di Zeus, gli uomini dell'Età d'Oro, divennero dei demoni, protettori degli esseri umani e dispensatori di beni, sempre invisibili e riconoscibili solo dalle loro azioni. 
1981), l'Agathos Daimon appare sotto forma di serpente, in un rilievo in calcare rinvenuto ad Akrai57, assieme alla sua controparte femminile, Agathé Tyche (Cfr. Herzog-Hauser, 1948; Szilàgyi, 1966; Villard, 1997), spogliata da tutti gli gli elementi ambigui della tradizione letteraria rappresentata come donna che indossa il polos e porta in mano una patera ed abbraccia una cornucopia, simbolo dell'abbondanza (fig. 13).

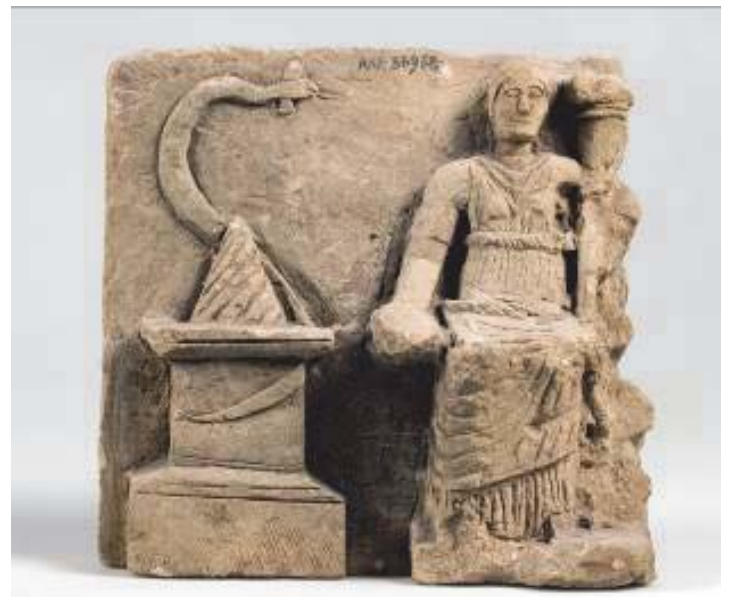

Fig. 13 - Rilievo in calcare con Agathos Daimon e Agathe Tyche (MAR Siracusa "Paolo Orsi")

Una piccola Sfinge fittile è presente nella collezione di terrecotte votive, provenienti da Akrai e conservate al Museo Archeologico Regionale "Antonio Salinas" di Palermo ${ }^{58}$. Dai tragediografi legata al mondo ctonio ${ }^{59}$, antagonista di Edipo nella saga tebaica60, la Sfinge ${ }^{61}$ è da molti riconnessa al senso stesso della Morte (Cfr. Herbig, 1929; Donadoni, 1966; Kourou, 1992; Kourou et alii 1997: 1165; Kourou, 2006). La sua immagine fu spessissimo usata fin dall'età micenea per accompagnare simbolicamente il defunto, rapito alla luce del Sole ed all'affetto dei suoi cari verso l'estrema dimora. Unica fra le antiche divinità pre-olimpiche ad essere sopravvissuta nella coscienza religiosa della società arcaica anche se decaduta al rango di mostro e come tale impossibile da propiziarsi (Cfr. da ultimo Papachatzis, 2006: 102-103), la Gorgone assunse un ruolo importante non solo in senso apotropaico ma anche cultuale (Cfr. Klingbeil, 1936. Contra Karagiorga, 1970: 133-134).

Diffusissimo elemento decorativo in quasi tutte le espressioni artistiche, il Gorgoneion, associato indissolubilmente alla dea Athena che la pose sulla sua egida, subì col passare dei secoli un'evoluzione anche nella sua rappresentazione (Cfr. Karagiorga, 1970).

Così si spiega ad esempio, come ornamento di un mobile ligneo, il busto di Medusa in bronzo ${ }^{62}$, appartenente tipologicamente alla seconda categoria di Furtwängler63, la così detta "bella Medusa", con un viso completamente umano e con l'unica aggiunta di un paio di alette sulle tempie (fig. 14).

57 II rilievo (MAR Sr 36968) può essere datato dopo il IV secolo a.C., quando il dio è rappresentato dal serpente accanto alla Agathe Tyche. Per la descrizione, cfr. Bernabò Brea 1956: 149, n. 20, tav. XXXII, 1.

${ }_{58}$ MAR Pa 883. Cfr. Distefano 2006: 58, n. 16, fig. 14.

${ }^{59}$ Esch., Sept., 539, 776-777 (simile a Kńp che rapisce gli uomini); Eur., Phoen., 810-811, 1019-1020.

60 Come mostra ad esempio, un cratere a campana a figure rosse (fine V secolo a.C.) appartenente alla Collezione Judica (n. 2805=85330), dove sul lato principale vi è la rappresentazione della sfida fra Edipo e la Sfinge. Cfr. Dibartolo 1996-2004: 143-144.

61 DEMGOL, s.v. Sfinge.

62 Donato al Museo Archeologico di Siracusa da Giuseppe Maria Amorelli, eletto da Papa Gregorio XVI a vescovo di Siracusa (3/2/183213/12/1840), il reperto fece parte fino al 1835 della collezione del barone Judica (Coll. Judica n. $2937=$ MAR Sr 94758). Per la sua datazione, cfr. Libertini 1929: 44, fig. 6 (datazione età ellenistica); Voretzsch 1957: 25, tav. VIII.3; Bonacasa 1984: 293 , fig. 342 (metà del II secolo a.C.) ed infine, Ciurcina 1996: 747, n. 371 (III secolo a.C.).

${ }^{63}$ Cfr. Furtwängler 1886-1890. Questo secondo tipo va datato al primo quarto del V secolo a.C., come dimostra anche la citazione di Pindaro (Pyth. XII, 16) che la definisce Eumapáou. 
Fig. 14 - Testina bronzea di Medusa (da Ciurcina 1996)

La torsione del busto che mostra chiari influssi di scuola ellenistica64, fa ritenere che la Gorgone si sia appena svegliata, un istante prima della morte che Perseo è venuto darle con il suo falcetto. Ritengo che l'artista abbia quindi tentato di fissare nel suo volto la visione della morte.

È da ricordare infine, un dato trasmesso dalla tradizione locale che si lega ancora inspiegabilmente con un toponimo tramandato dalle epigrafi.

Ai piedi del versante meridionale dell'Acremonte, lungo la trazzera che porta ancor oggi al contado, si trovano i c.d. Monti Allegri («Muntalleri»), due piccoli cocuzzoli artificiali che la ricerca dotta locale ha identificato con le c.d. Mammelle di Lamia (Cfr. Bernabò Brea, 1956: 29-30), citate dalla famosa epigrafe edita da Kaibel65 in cui è esposta per la concessione di affitti di themelia per la panegyris locale (Cfr. Manganaro, 1996-2004), e grazie alla quale si conoscono alcuni elementi di topografia urbana della Akrai ellenistica.

Quale sia il nesso del toponimo con il demone femminile che mangiava bambini66 è difficile a dirsi ${ }^{67}$.

\section{Conclusioni}

Da quanto si è potuto esaminare appare certo che ad Akrai, se da un lato la figura rassicurante di Herakles, unico eroe immune dalla punizione divina per la sua hybris (Cfr. Brelich, 2010: 209), nella sua duplice funzione di oikistes e di phylakterion fu da subito assunto a modello per la salvezza della giovane sub-colonia già in età arcaica, con lo scorrere vorticoso degli anni ed il cambio drastico e repentino nella guida della metropoli, si ebbe anche un riflesso nella vita quotidiana nella periferia dell'epicrazia siracusana, con l'adozione di un modello soteriologico di stampo ellenistico.

Ecco che la trasformazione in luogo di culto della latomia posta sul versante sud-orientale dell'Acremonte, agli inizi del III secolo a.C., assolse al duplice scopo perseguito poi da lerone II, di ruolo al contempo civile e religioso. Questi Heroa dove gli Acrensi in età ellenistica rendevano onore ai defunti eroizzati (dei veri e propri "caduti sul campo") permisero una maggiore coesione sociale, un più forte legame con la metropoli (e di rimando con lo stesso basileus) ed infine una risposta alle istanze impellenti sulla sfera del post mortem che si propagava ineluttabilmente in tutto il bacino del Mediterraneo.

II ruolo del pantheon olimpico in una piccola polis dell'ultimo regno ellenistico d'Occidente non fu solo quello di integrare la società siceliota nell'alveo dell'Ellenismo metropolitano ma anche quello di garante dell'ordine costituito, aldilà delle aspre lotte che insanguinavano l'isola nel III secolo a.C.

Spostando l'attenzione al versante meno benevolo della concezione ultraterrena antica, alcune figure mostruose (Sfinge, Medusa, etc.) legate all'Ade che appaiono più o meno frequentemente nell'iconografia locale (sia fittile che

\footnotetext{
64 Distefano (1995) la mette in relazione anche con il frammento vitreo con raffigurazione di Iside, rinvenuto sulle pendici meridionali dell'Acremonte.

${ }^{65}$ IG, XIV, 217.

${ }^{66}$ Figlia di Belo, re della Libia, fu amata da Zeus ma a causa della gelosia di Hera, perse tutti i suoi figli ad eccezione di Scilla. Da allora, tramutatasi in mostro, si nascose in una grotta e si vendicò uccidendo e divorando i figli altrui. Sull'etimologia del nome, cfr. DEMGOL, s.v. Lamia.

${ }^{67}$ Per una breve carrellata sulle tradizioni popolari locali cfr. Blancato 2012. Sul legame degli spauracchi soprattutto femminili con la sfera dell'educazione infantile, cfr. Pellizer 1998.
} 
ceramica), furono usate in maniera apotropaica.

Se la nostra analisi cogliesse nel giusto, si assisterebbe insomma, al tentativo di sondare le due facce della stessa medaglia attraverso la documentazione (certo frammentaria ed ancora incompleta) archeologica: l'Aldilà è popolato da spiriti ed ombre non sempre benigne all'uomo, come insegna il brano della Nekyia di Omero. Ė perciò ritenuto un dovere ineludibile della religiosità pagana (e greca in particolare) quello di placare le forze distruttive della natura ed al contempo, di purificare l'ambiente circostante per ottenere una vita terrena il meno possibile afflitta da mali e sventure.

\section{Bibliografia}

Agnello 1965: G. Agnello, "Gabriele Judica e le fortunose vicende del suo museo", ArchStorSir 11, 1965, pp. 78-136.

Ampolo 2011: C. Ampolo (a.c.d.), Siracusa. Immagine e storia di una città, Pisa 2011.

Antonaccio 1995: C.M. Antonaccio, An Archaeology of Ancestors. Tomb Cult and Hero Cult in Early Greece, Lanham 1995.

Bell 1999: M. Bell, "Centro e periferia nel regno siracusano di lerone II", in G. Vallet - E. Greco (eds.), "La colonisation Grecque en Méditerranée Occidentale». Actes de la rencontre scientifique en hommage a George Vallet, organisée par le Centre Jean Bérard, l'Ecole Française de Rome, l'Istituto Universitario Orientale et l'Università degli studi di Napoli "Federico II", (Rome Naples, 15-18/11/1995), Roma 1999, pp. 257-277.

Bermond Montanari 1958: G. Bermond Montanari, s.v. Agathodaimon, in EAA, I, pp. 134-135.

Bernabò Brea 1956: L. Bernabò Brea, Akrai [Società di Storia Patria per la Sicilia Orientale. S. III, Monografie Archeologiche della Sicilia, I], Catania 1956.

Bernabò Brea 1986: L. Bernabò Brea, II tempio di Afrodite ad Akrai [Cahiers du Centre Jean Bérard, X], Napoli 1986.

Blancato 2012: N. Blancato, "Tra mitologia e credenza popolare: gli incantesimi di "Montalleri» e gli spiriti di "Quagghialatti»" (http://nelloblancato.blogspot.com/2012/04/).

Blanck 2002-2003: H. Blanck, Giovani archeologi tedeschi nel Ragusano e nel Siracusano, in P. Pelagatti - G. Distefano - L. De Lachenal (a cura di), "Camarina 2600 anni dopo la fondazione». Nuovi studi sulla città e sul territorio. Atti del Convegno Internazionale (Ragusa, 7/12/2002 e 7-9/4/2003), Roma 2006, pp. 313-326.

Bonacasa - Joly 1984: N. Bonacasa - E. Joly, L'Ellenismo e la tradizione Ellenistica, in G. Pugliese Carratelli (a cura di), Sikanie. Storia e civiltà della Sicilia greca [Antica Madre,VIII], Milano 1984, pp. 277-358.

Brelich 20102: A. Brelich, Gli eroi greci: un problema storico-religioso, Roma 1958 (Milano 20102).

Burkert 1977: W. Burkert, Griechische Religion der archaischen und klassischen Epoche [Die Religionen der Menschheit, 15], Stuttgart 1977 [AӨńvaı 1993].

Burkert 1979: W. Burkert, Structure and History in Greek Mythology and Ritual. Berkeley 1979 [AӨńvaı 19972].

Burkert 19972: W. Burkert, Homo Necans. Interpretationen altgriechischer Opferriten und Mythen [Religionsgeschichtliche Versuche und Vorarbeiten, 32], Berlin 1972 (19972) [AӨńvaı 2011].

Coarelli 1984: F. Coarelli, s.v. Akrai, in F. Coarelli - M. Torelli, Sicilia, Roma-Bari 1984.

Consolo Langher 2000: S.N. Consolo Langher, Religione e Regalità. Tra Grecia, Oriente e Sicilia: fondamenti ideologici e politici nel culto del sovrano ellenistico, in P. Anello - G. Martorana - R. Sammartano (a cura di), "Ethne e religioni nella Sicilia antica». Atti del Convegno (Palermo, 6-7/12/2000) [Kokalos. Supplemento, XVIII], Roma 2006, pp. 329-342.

Conze 1880: A. Conze, Hermes Kadmilos, AZ 38, 1880, pp. 1-10.

Copani 2008: F. Copani, Acre e Casmene. L'espansione siracusana sui Monti Iblei, in G. Zanetto - M. Ornaghi (a cura di), Argumenta antiquitatis. Seminari 2008 [Acme. Quaderni, CIX], Milano 2009, pp. 11-21.

Cordiano 1997: G. Cordiano, La Ginnasiarchia nelle «polis» di Occidente nel Mediterraneo Antico, Pisa 1997.

Cuartero i Iborra 1998: F.J. Cuartero i lborra, Hèracles, fundador de sacrificis: l'heroi i les tres funcions, Faventia 20, 2, 1998, pp. 15-25.

De Miro 1966: E. De Miro, Latomia con incavi votivi in località Campo Sportivo, BA 51, 1966, pp. 90-91.

De Miro 1981: E. De Miro, Civiltà rupestre dell'Agrigentino. Esempi dalla Preistoria al Medioevo, in C.D. Fonseca (a cura di), «La Sicilia Rupestre nel contesto della Civiltà Medioevale». Atti del VI Convegno Internazionale di Studio sulla Civiltà rupestre Medioevale nel Mezzogiorno d'Italia (Catania-Pantalica-Ispica, 7-12/9/1981) [Saggi e ricerche, 18], Galatina 1986, pp. 235-244.

De Sensi Sestito 1977: G. De Sensi Sestito, Gerone II. Un monarca ellenistico in Sicilia [KLEIO, I], Palermo 1977.

Dibartolo 1996-2004: L. Dibartolo, Gabriele Judica: lampadoforo del passato, Studi Acrensi 3, 1996-2004, pp. 119-150.

Dimartino 2003: A Dimartino, Per una revisione dei documenti epigrafici siracusani pertinenti al regno di lerone II, in C. Michelini (a cura di), «Guerra e pace in Sicilia e nel Mediterraneo antico (VIII-III sec. a.C.). Arte, prassi e teoria della pace e della guerra». Atti delle quinte giornate internazionali di studi sull'area elima e la Sicilia occidentale nel contesto mediterraneo (Erice, 1215/10/2003) [Seminari e Convegni, 7], II, Pisa 2006, pp. 703-717.

Distefano G. 2000: G. Distefano, Un rilievo votivo con banchetto funerario da Acrillae, SicA 33, 98, 2000, pp. 277-281.

Distefano S. 1995: S. Distefano, Le origini del culto di Iside ad Akrai, Prospettive Siracusa 14, 4, pp. 48-51.

Distefano S. 2006: S. Distefano, Le terracotte della collezione acrense del museo A. Salinas di Palermo: Contributo allo studio dei culti e delle istituzioni religiose di Akrai, Catania 2006.

Distefano S. 2009: S. Distefano, Jerone II, re degli Acrensi. Contributo alla storia di Akrai tra le due guerre puniche, in I cinquant'anni del Platone, Canicattini Bagni 2009, pp. 214-261.

Di Vita 1956: A. Di Vita, La penetrazione siracusana nella Sicilia sud-orientale alla luce delle più recenti scoperte archeologiche, Kokalos 


\section{2, 2, 1956, pp. 177-205.}

Donadoni 1966: S. Donadoni, s.v. Sfinge, in EAA, VII, pp. 231-232.

Durrand 1981: F. Durand, s.v. Agathodaimon, in LIMC, I', pp. 277-282 e l², pp. 203-207, nn. 1-40.

Ekroth 2002: G. Ekroth, The sacrificial rituals of Greek hero-cults in the Archaic to the early Hellenistic periods [Kernos, Suppl. 12], Liège 2002.

Furtwängler 1890: A. Furtwängler, s.v. Gorgones und Gorgo, in Roscher ML, I.2, 1890, pp. 1695-1728.

Gansichinietz 1918: R. Gansichinietz, s.v. Agathodaimon (1), in RE, S. III, 1918, cc. 37-59.

Gauthier 1995: P. Gauthier, Notes sur le rôle du gymnase dans les cites hellénistiques, in M. Wörrle - P. Zanker (hrsg.), Stadtbild und Bürgerbild im Hellenismus, München 1995, pp. 1-11.

Germanà Bozza 2008: G. Germanà Bozza, Da ippeis ad equites: osservazioni sull'iconografia di alcuni rilievi funerari siracusani del III secolo a.C., Vexillum 3, 2008, pp. 4-14.

Guarducci 1962: M. Guarducci, Bryaktes. Un contributo allo studio dei «banchetti eroici», AJA 66, 3, 1962, pp. 273-280. [Ristampato in Scritti scelti sulla religione greca e romana e sul cristianesimo, Leiden 1983, pp. 10-19]

Guzzetta 2002: G. Guzzetta, s.v. Acrae, in BNP, I, c. 108.

Herbig 1929: R. Herbig, s.v. Sphinx, in RE, III A², cc. 1704-1749.

Herzog-Hauser 1948: G. Herzog-Hauser, s.v. Tyche (1), in RE, VII A², cc. 1643-1689.

Hülsen 1893: Ch. Hülsen, s.v. Akrai, in RE, I' c. 1192.

Icard-Gianolio 2000: N. Icard-Gianolio, Héraclès fondateur, in Agathos Daimôn, Mythes et Cultes. Etudes d'iconographie en l'honneur de Lilly Kahil [BCH Suppl. 38], Athènes, pp. 219-228.

Jourdain-Annequin 2006: C. Jourdain-Annequin, Héraklès en Occident, in A. Coppola (a cura di), «Eroi, eroismi, eroizzazioni»: dalla Grecia antica a Padova e Venezia. Atti del Convegno Internazionale (Padova, 18-19/9/2006), Padova, 2007, pp. 5-16.

Judica 1819: G. Judica, Le antichità di Acre scoperte, descritte ed illustrate dal Barone Gabriele Judica, Messina 1819.

Kerényi 1944: K. Kerényi, Hermes, der Seelenführer [Albae Vigiliae, 1], Zürich 1944. [Guide of Souls, Woodstock 19966]

Kerényi 19523: K. Kerényi, Die antike Religion, Düsseldorf 1952³. [Religione antica, Milano, 2001].

Klingbeil 1936: W. Klingbeil, Kopf-Masken und Maskierungszauber in den antiken Hochkulturen insbesondere des Alten Orients, Berlin 1936

Kourou 1992: N. Kourou, Aegean Orientalizing versus Oriental Art: the Evidence of Monsters, in V. Karageorghis (ed.), The Civilizations of the Aegean and their Diffusion in Cyprus and the Eastern Mediterranean, 2000-600 B.C, Larnaca 1992, pp. 110-123.

Kourou 2006: N. Kourou, Following the Sphinx. Tradition and Innovation in Early Iron Age Crete, in G. Rizza (a cura di), «ldentità culturale, etnicità, processi di trasformazione a Creta fra Dark Age e Arcaismo». Per i cento anni dello scavo di Priniàs (19062006). Atti del Convegno di Studi (Atene, 9-12/11/2006) [Studi e Materiali di Archeologia Greca, 10], Palermo 2011, pp. $165-177$.

Kourou et alii 1997: N. Kourou et alii, s.v. Sphinx, in LIMC, VIII', pp. 1149-1174.

Krauskopf - Dahlinger 1988: I. Krauskopf - S.C. Dahlinger, s.v. Gorgo, Gorgones, in LIMC, IV1 ${ }^{1}$ pp. 285-330.

Kunz 2006: H. Kunz, Sicilia. Religionsgeschichte des römischen Sizilien [Religion der Römischer Provinz, 4], Tübingen 2006.

La Rosa 1988-1989: V. La Rosa, Per la Neaiton ellenistica: un saggio di scavo nella zona del ginnasio, in AMISVNA, 19-20, 1988-1989, pp. 75-104.

La Rosa 1993: V. La Rosa, s.v. Noto - Storia della ricerca archeologica (con L. Arcifa), in BTCGI, XII, Pisa-Roma 1993, pp. 410-412.

La Torre 2008: G.F. La Torre, Dal phrourion alla Polis. Città d'altura nella Sicilia ellenistica, in M. Congiu - C. Miccichè - S. Modeo (a cura di), «EIS AKPA». Insediamenti d'altura in Sicilia dalla Preistoria al III sec. a.C. Atti del V Convegno di Studi (Caltanissetta, 10-11/5/2008) [Triskeles, V], Caltanissetta 2009, pp. 191-204

Lehmler 2005: C. Lehmler, Syrakus unter Agathokles und Hieron II. Die Verbindung von Kultur und Macht in einer hellenistischen Metropole, Frankfurt 2005.

Lévêque - Verbanck-Piérard 1992: P. Lévêque - A. Verbanck-Piérard, Héraclès. Héros ou dieu?, in C. Bonnet - C. Jourdain-Annequin (éds.), Héraclès. D’une rive à l'autre de la Méditerranée, Bruxelles-Rome 1992, pp. 263-291.

Libertini 1929: G. Libertini, II Regio Museo archeologico di Siracusa, Roma 1929.

Lombardo L. 1998: L. Lombardo, Gabriele Judica e gli scavi di Acre, ArchStorSir, s. III, 12, pp. 169-214.

Lombardo M. 2006: M. Lombardo, Da apoikiai a metropolis. Dal progetto al convegno, in M. Lombardo - F. Frisone (a cura di), «Colonie di colonie». Le fondazioni sub-coloniali greche fra colonizzazione e colonialismo. Atti del Convegno (Lecce, 22-24/6/2006) [Collana del Dipartimento di Beni Culturali dell'Università del Salento, 16], Galatina 2009, pp. 17-30.

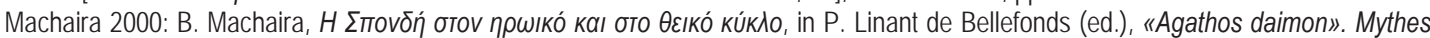
et cultes: études d'iconographie en I'honneur de Lilly Kahil [BCH, Suppl. 38], Athènes-Paris 2000, pp. 339-344.

Machaira et alii 1992: B. Machaira et alii, s.v. Heros Equitans, in LIMC, VI¹, pp. 1019-1081.

Manganaro 1996-2004: G. Manganaro, Affitto di spazi pubblici nel contesto urbano di Akrai, Studi Acrensi 3, 1996-2004, pp. 1-16.

Manganaro 2005: G. Manganaro, La mazza di Herakles, Epigraphica 67, 1-2, 2005, pp. 9-16.

Musumeci 2009: M. Musumeci, Gabriele Judica, le sue ricerche e la collezione Judica, in A. Crispino - A. Musumeci (a cura di), «Musei nascosti». Collezione e raccolte archeologiche a Siracusa dal XVII al XX secolo. Catalogo della mostra (Siracusa, 6/12/2008 15/2/2009), Napoli 2009, pp. 35-41.

Nilsson 1906: M.P. Nilsson, Griechische Feste von religiöser Bedeutung mit Ausschluss der Attischen, Leipzig 1906 (1995³).

Nilsson 1940: M.P. Nilsson, Greek Popular Religion, New York 1940. [AӨńvaı 2006³].

Nilsson 19492: M.P. Nilsson, A History of Greek Religion, Oxford 19492. [AӨńvaı 2008¹]. 
Nilsson 19502: M.P. Nilsson, The Minoan-Mycenaean Religion and its Survivals in Greek Religion [Acta Regiae Societatis humaniorum litterarum Lundensis, IX], Lund $1950^{2}$.

Orsi 1897: P. Orsi, Noto Vecchio (Netum). Esplorazioni archeologiche, NSc, s. IV, pp. 69-90.

Orsi 1904: P. Orsi, Siracusa, NSc, s. V, 1, pp. 275-291.

Orsi 1931: P. Orsi, Studi preliminari sulla topografia dell'antica Enna, NSc, s. VI, 7, pp. 373-394.

Otto 19473: W. Otto, Die Götter Griechenlands. Das Bild des Göttlichen im Spiegel des griechischen Geistes, Tübingen 19473 [Milano 2004].

Pace 1945: B. Pace, Arte e civiltà della Sicilia antica, III, Roma 1945.

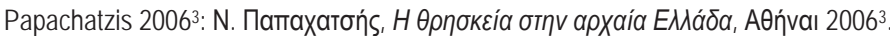

Pedrucci 2009: G. Pedrucci, Cibele, Frigia e la Sicilia, Roma 2009.

Pellizer 1998 : E. Pellizer, Figures de croquemitaines féminins en Grèce antique, MAR 2-4, 1998, pp. 141-151.

Polacco - Mirisola 2005: L. Polacco - R. Mirisola, II santuario siracusano delle cento are, QuadMed 13, 2005, pp. 15-36.

Portale 2010: E.C. Portale, "Iconografia funeraria" e pratiche devozionali nella Sicilia ellenistica: il "Totenmahl", Sicilia Antiqua 7, 2010, pp. $39-78$

Pugliese Carratelli 1996: G. Pugliese Carratelli (a cura di), I Greci in Occidente, Milano 1996.

Raingeard 1935: P. Raingeard, Hermès Psychagogue. Essai sur les origines du culte d'Hermès, Paris 1935.

Ross Taylor 1927: L. Ross Taylor, The 'Proskynesis' and the Hellenistic Ruler Cult, JHS 47, 1, 1927, pp. 53-62.

Ross Taylor 1930: L. Ross Taylor, Alexander and the Serpent of Alexandria, CIPh 25, 4, 1930, pp. 375-378.

Schubring 1867: J. Schubring, Akrä-Palazzolo. Eine topographisch-archäologische Skizze, JKPh, Suppl. IV, 8, 1867, pp. 659-672.

Scirpo 1996-2004: P.D. Scirpo, Bibliografia generale su Akrai: 1537-2002, Studi Acrensi 3, 1996-2004, pp. 213-295.

Scirpo 2004: P.D. Scirpo, Un esempio sul rapporto delle sub-colonie siceliote con la metropolis: Syrakousai-Akrai, Diachronia 6, 2004, pp. 23-32.

Scirpo 2005: P.D. Scirpo, Sui culti delle sub-colonie arcaiche di Siracusa, in P.D. Scirpo (a cura di), TRISKELES [Diachronia, Suppl. 1], Atene 2005, pp. 49-58.

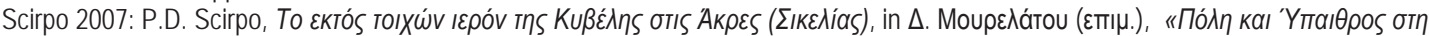

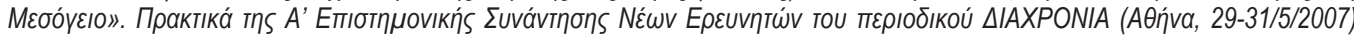
[Diachronia, Suppl. 2], AӨńvaı 2012, pp. 63-78.

Scirpo 2010: P.D. Scirpo, The Founder-Cult of Hieron II at Akrai: The Rock-Relief from Intagliatella's Latomy, in S.A. Paipetis - M. Ceccarelli (eds.), The Genius of Archimedes - 23 Centuries of Influence on Mathematics, Science, and Engineering [History of Mechanism and Machine Science, 11], Dordrecht 2010, pp. 429-438.

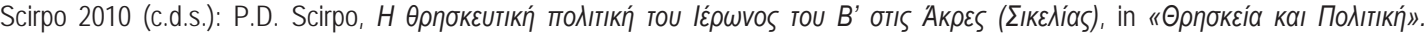

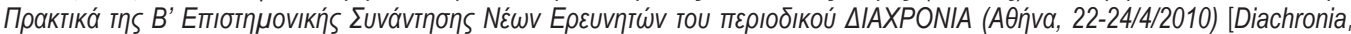
Suppl. 4], AӨńvaı, c.d.s.

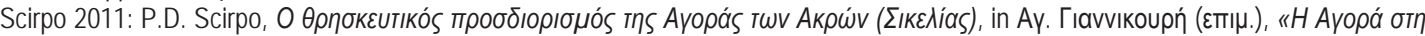

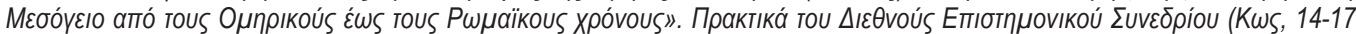
Aтріגíou 2011), AӨńva 2011, pp. 61-70.

Scirpo 2005-2013: P.D. Scirpo, Bibliografia generale su Akrai. Addenda e Corrigenda, Studi Acrensi 4, 2005-2013, pp. $150-172$.

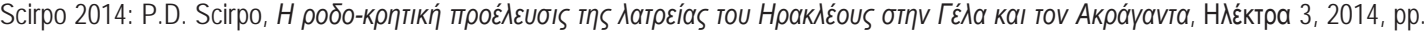
65-87.

Scirpo 2015 (c.d.s.): P.D. Scirpo, Brevi note sul santuario rupestre di Kybele ad Akrai, QuadMed 15, 2015, c.d.s.

Seiffert 2005: A. Seiffert, s.v. Heroon, in ThesCRA, IV, pp. 24-38.

Sfameni Gasparro 1973: G. Sfameno Gasparro, I culti orientali in Sicilia [EPRO, 31], Leiden 1973.

Sfameni Gasparro 1997: G. Sfameni Gasparro, Daimôn and Tychê in the Hellenistic Religious Experience, in L. Hannestad - J. Zahle T. Engberg-Pedersen - P. Bilde (eds.), Conventional Values of the Hellenistic Greeks [Studies in Hellenistic Civilization, VIII], Aarhus 1997, pp. 67-109.

Simon 2004: E. Simon, s.v. Libation, in ThesCRA, I, pp. 237-253.

Stafford 2012: E. Stafford, Herakles [Gods and Heroes in the Ancient World, 10], London 2012.

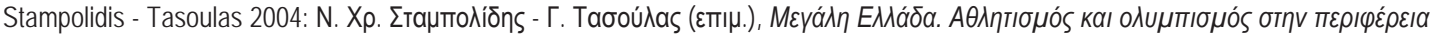

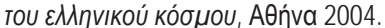

Szilàgyi 1966: G.J. Szilàgyi, s.v. Tyche, in EAA, VII, pp. 1038-1041.

Trümpy 1997: C. Trümpy, Untersuchungen zu den altgriechischen Monatsnamen und Monatsfolgen [Bibliothek der klassischer Altertumswissemschaften, Reihe II, N.F., b. 98], Heidelberg 1997.

Tusa 1958: V. Tusa, s.v. Acre, in EAA, I, pp. 46-48.

Villard 1997: L. Villard, s.v. Tyche, in LIMC, VIII' , pp. 115-125 e VIII2, pp. 85-109, nn. 1-197.

Voretzsch 1957: E.A. Voretzsch, Ein römisches Porträt-Medaillon in Afghanistan, RM 64, 1957, pp. 8-45.

Voza 1971: G. Voza, Akrai, in G. Voza - P. Pelagatti (a cura di), Un quinquennio di attività archeologica nella provincia di Siracusa, Siracusa 1971, pp. 72-74, tav. XXI.

Voza 1973: G. Voza, Akrai, in P. Pelagatti - G. Voza (a cura di), Archeologia nella Sicilia sud-orientale, Napoli 1973, pp. 127-128.

Voza 1976: G. Voza, s.v. Akrai, in PECS, pp. 26-27.

Voza 1980a: G. Voza, s.v. Akrai, in E. Gabba - G. Vallet (a cura di), La Sicilia antica, l33, Napoli 1980, pp. 497-507. 
Voza 1980b: G. Voza, Nuove ricerche a Siracusa, Akrai, Monte Casale ed Eloro, in «Architettura ed Urbanistica nella Sicilia Greca Arcaica». Atti della III Riunione scientifica della Scuola di perfezionamento in Archeologia Classica dell'Università di Catania (Siracusa, 11-14/12/1980) [CronA 19, (1980)], pp. 69-71.

Voza 1999: G. Voza, Nel segno dell'antico. Archeologia nel territorio di Siracusa, Palermo 1999.

Voza - Lanza 1971-1994: G. Voza - M.T. Lanza, s.v. Acre, in EAA, Suppl.2, I, pp. 38-39.

Ziegler 1964: K. Ziegler, s.v. Akrai, in KP, I, c. 221. 